\title{
Genetic structure and historical demography of Malus sieversii in the Yili Valley and the western mountains of the J unggar Basin, Xinjiang, China
}

\author{
HongXiang ZHANG ${ }^{1}$, MingLi ZHANG ${ }^{1,2 *}$, LiNa WANG ${ }^{3}$ \\ ${ }^{1}$ Key Laboratory of Biogeography and Bioresource in Arid Land, Xinjiang Institute of Ecology and Geography, Chinese Academy of \\ Sciences, Urumqi 830011, China; \\ ${ }^{2}$ Institute of Botany, Chinese Academy of Sciences, Beijing 100093, China; \\ ${ }^{3}$ Central China Normal University, Wuhan 430079, China
}

\begin{abstract}
Malus sieversii, a wild progenitor of domesticated apple, is distributed in western Xinjiang of China, eastern part of Kazakhstan and Kyrgyzstan in Central Asia. To well understand the genetic structure and the historical demography of this important germplasm resource, we sampled 15 populations with 110 individuals of Malus sieversii from the Yili Valley and the western mountains of the Junggar Basin, Xinjiang, and sequenced two nrDNA fragments for these 110 individuals. Meanwhile, we modeled and compared species distributions under the current and the Last Glacial Maximum climatic conditions. The results showed that populations of $M$. sieversii from Xinjiang had low levels of genetic diversity and genetic differentiation. During the LGM period, populations of $M$. sieversii had lost their northern distributions in the western mountains of the Junggar Basin. M. sieversii has experienced a demographic expansion from the south of the Yili Valley to the north of the western mountains of the Junggar Basin during the warm interglacial epochs. Due to the high sensibility of $M$. sieversii to disturbance, we proposed more attention should be paid to the $M$. sieversii populations in the western mountains of the Junggar Basin.
\end{abstract}

Keywords: Malus sieversii; phylogeography; LEAFY gene intron 1; species distribution modeling; genetic diversity

Citation: HongXiang ZHANG, MingLi ZHANG, LiNa WANG. 2015. Genetic structure and historical demography of Malus sieversii in the Yili Valley and the western mountains of the Junggar Basin, Xinjiang, China. Journal of Arid Land, 7(2): 264-271. doi: 10.1007/s40333-014-0044-2

The distribution of Malus sieversii covers western Xinjiang in China, eastern part of Kazakhstan and Kyrgyzstan in Central Asia. The molecular phylogeny of genus Malus shows the cultivated apple (M. domestica) has a close relationship with M. sieversii (Zhou and $\mathrm{Li}, 2000$; Robinson et al., 2001; Harris et al., 2002). The wild apple in Central Asia, M. sieversii, contributes a lot to the cultivated apple and is a main progenitor of domesticated apple. Because of the high value in potential cultivar breeding of apple, $M$. sieversii has recently attracted many interests from botanists in investigating the genetic diversity and the population structure.

Using a few types of molecular markers, such as
Simple Sequence Repeat (SSR) and Sequence-related Amplified Polymorphism (SRAP) analysis, $M$. sieversii populations in western Xinjiang have low level of genetic differentiations between populations (Zhang et al., 2007; Zhang et al., 2009). And the genetic variations mainly occurred within populations. It also shows higher levels of genetic diversity in southern populations of M. sieversii in Yili Valley. Although populations in Yili Valley have close relationships, the different geographical populations could be relatively independent populations based on genetic clustering results (Zhang et al., 2007, 2009). Yan et al. (2008) has also surveyed the variations in distribution, pollen exine and Random Amplified Polymorphic DNA

\footnotetext{
"Corresponding author: MingLi ZHANG (E-mail: zhangml@ibcas.ac.cn) Received 2014-03-04; revised 2014-09-02; accepted 2014-11-06

(C) Xinjiang Institute of Ecology and Geography, Chinese Academy of Sciences, Science Press and Springer-Verlag Berlin Heidelberg 2015
} 
(RAPD) polymorphisms of $M$. sieversii. The results elaborated the biological diversity of $M$. sieversii and the relationship to ecological environment. Similarly, the genetic structure in western distribution of $M$. sieversii, including Kazakhstan and Kyrgyzstan in Central Asia, was also studied using the method of molecular markers (Lamboy et al., 1996; Richards et al., 2009). Molecular variances of $M$. sieversii mostly appeared within populations in Kazakhstan and Kyrgyzstan. Level of genetic differentiation was low between populations, while the southern locations of $M$. sieversii populations had high level of genetic diversity.

$M$. sieversii is a major component of wild fruit forest in the Central Asian Mountains. Wild fruit forest is a "relict" of subtropical broad-leaved forest in the Early Tertiary (Zhang, 1973; Hou and Xu, 2005; Sitpaeva and Chekalin, 2013). Most subtropical broadleaved species could have undergone southward migrations during the process of enhanced aridity along with the retreat of the Tethys. Due to the humid and temperate climate, wild fruit forests have occurred in some valley and middle altitude zone of the Tianshan Mountains. The Central Asian Mountains have been experienced multiple glaciations during the Quaternary evidencing from the ancient glaciers tracks (Wen and Shi, 1993; Xu et al., 2010). These Quaternary glaciations should have intensively affected the distribution of wild fruit forests in the Tianshan Mountains
(Zhang, 1973; Hou and Xu, 2005). In our previous studies, we proposed the western region of the Tianshan Mountains, such as the Yili Valley, could provide refugee for plant species to growth during the cold-dry glacial periods (Zhang and Zhang, 2012; Zhang et al., 2013).

At present, $M$. sieversii is mainly distributed in the refugee region of the Tianshan Mountains. The current distribution pattern and the historical demography of $M$. sieversii have not been studied so far. Here, we aim to investigate the population genetics and the phylogeographical structure of $M$. sieversii using the methods of nrDNA sequences and species distribution modeling. In addition, we want to propose some advices on conservation management of this important germplasm resource.

\section{Materials and methods}

\subsection{Population sampling}

A total of 15 populations with 110 individuals of $M a-$ lus sieversii were collected from the Yili Valley of Tianshan Mountains and the western mountains of the Junggar Basin (Table 1; Fig. 1). We collected their fresh leaves, then dried and stored in silica gel in field. Voucher specimens for all populations were deposited in the Herbarium of Xinjiang Institute of Ecology and Geography, Chinese Academy of Sciences (XJBI).

Table 1 Population/group names, number of individuals sampling $\left(N_{\text {ind }}\right)$, allele distribution and estimation of unbiased expected heterozygosity $\left(\mathrm{H}_{\mathrm{e}}\right)$ for each population of Malus sieversii

\begin{tabular}{ccll}
\hline \multicolumn{1}{c}{ Population/Group } & $N_{\text {ind }}$ & \multicolumn{1}{c}{$\mathrm{H}_{\mathrm{e}}$} & \multicolumn{1}{l}{ Alleles } \\
\hline Western mountains of the Junggar Basin & 61 & 0.308 & \\
\hline EM-MFG & 16 & 0.175 & $1 / 1(13) ; 1 / 2(3)$ \\
EM-GXG & 6 & 0.409 & $1 / 1(4) ; 2 / 2(1) ; 1 / 2(1)$ \\
EM-DN & 6 & 0.485 & $1 / 1(2) ; 1 / 2(4)$ \\
EM-YZG & 3 & 0.000 & $1 / 1(3)$ \\
EM-SMG & 9 & 0.000 & $1 / 1(9)$ \\
TL-GZG1 & 5 & 0.356 & $1 / 1(4) ; 2 / 2(1)$ \\
TL-GZG2 & 7 & 0.440 & $1 / 1(3) ; 1 / 2(4)$ \\
TL-GZG3 & 9 & 0.503 & $1 / 1(5) ; 2 / 2(3) ; 1 / 2(1)$ \\
\hline Yili Valley & 49 & 0.488 & $1 / 1(2) ; 1 / 2(5)$ \\
XY-JTH1 & 7 & 0.495 & $1 / 1(4) ; 2 / 2(1) ; 1 / 2(4)$ \\
XY-JTH2 & 9 & 0.471 & $1 / 1(7) ; 2 / 2(1) ; 1 / 2(1)$ \\
GL-MHE1 & 9 & 0.294 & $1 / 1(1) ; 2 / 2(1)$ \\
GL-SW & 2 & 0.667 & $1 / 1(4) ; 2 / 2(2) ; 1 / 2(1)$ \\
GL-MHE1 & 7 & 0.495 & $1 / 1(1) ; 2 / 2(3) ; 1 / 2(4)$ \\
HC-ET & 8 & 0.500 & $1 / 1(2) ; 2 / 2(4) ; 1 / 2(1)$ \\
HC-GZG & 7 & 0.495 &
\end{tabular}




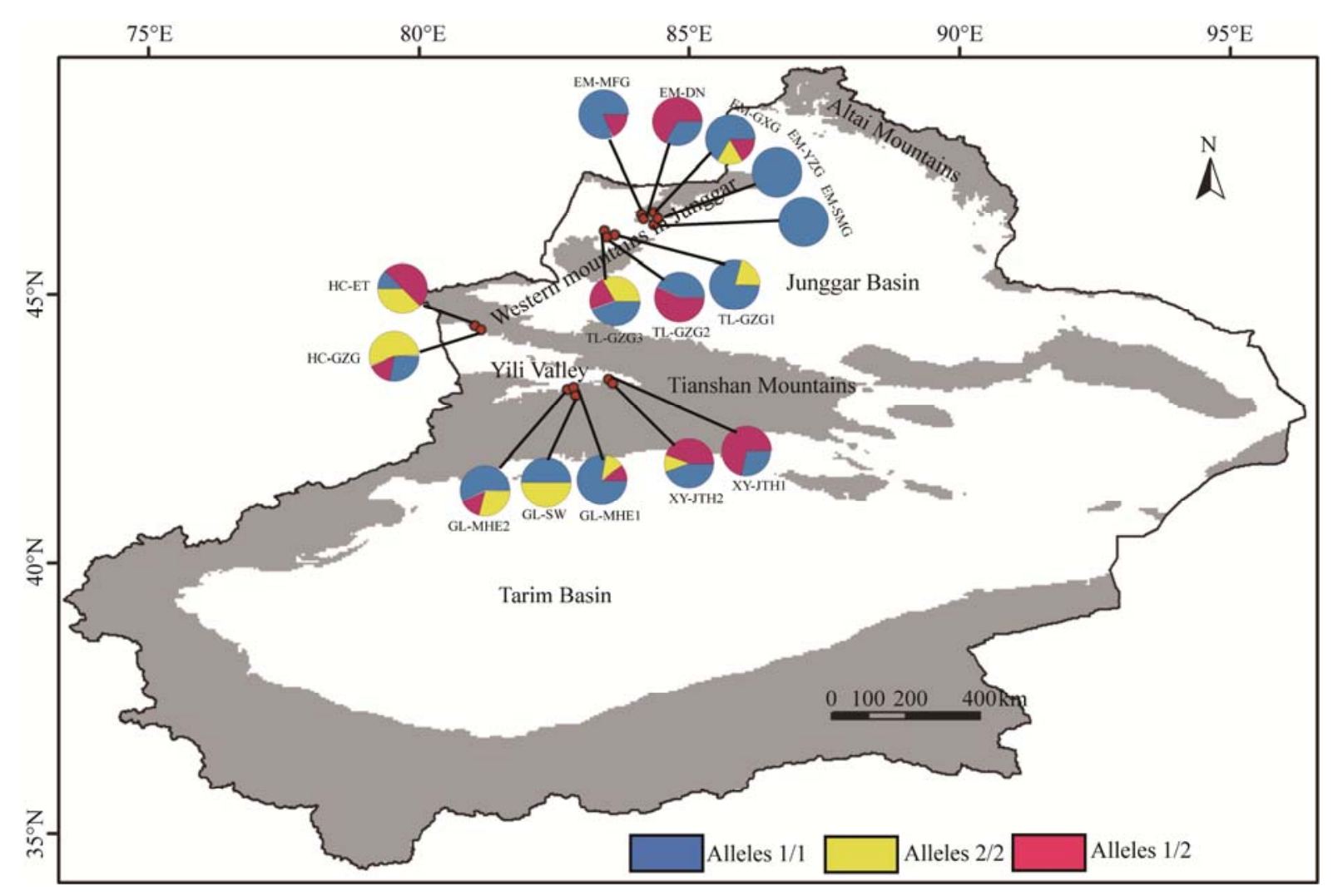

Fig. 1 Sampling locations and alleles distribution of Malus sieversii from the Yili Valley and the western mountains of the Junggar Basin

\subsection{DNA extraction, amplification and sequencing}

Total genomic DNA was extracted from approximately $50 \mathrm{mg}$ silica gel-dried leaf material per sample, following a CTAB protocol modified from Doyle and Doyle (1987). For obtaining adequate nucleotide variations, we selected more than ten pairs of chloroplast primer, such as $p s b \mathrm{~A}-t r n \mathrm{H}$, trnS-trnG, trnL-trnF, $a t p \mathrm{~B}-r b c \mathrm{~L}, \quad r p l 20-r p s 12, \quad p s b \mathrm{~B}-p s b \mathrm{H}, \quad r p s 16-t r n \mathrm{Q}$, $n d h F-r p l 32$, trnD-trnT, rpl16 and ycf6-psbM (Shaw et al., 2005, 2007). However, sequence variations were not identified in these cpDNA regions. Then, we tried to select nuclear markers. At last, nuclear regions of the ITS (Zhang et al., 2013) and the intron 1 of the LEAFY gene (Lo et al., 2009) were amplified and sequenced. The polymerase chain reaction (PCR) mixture and amplification program followed the protocols of Zhang and Zhang (2012). The PCR products were purified from an agarose gel using the PCR product purification kit following the recommended protocol (Sangon Biotech, Shanghai, China). Sequencing reactions were carried out, with the primers described above, in both directions by standard methods, on an ABI 3730 automated sequencer at the la- boratories of Sangon Biotech (Shanghai, China). We aligned these nucleotide sequences using CLUSTALX (Thompson et al., 1997) then checked them manually. Based on the chromatograms of both reading directions of sequences, we detected three additive nucleotides in each of nrITS sequence and an additive nucleotide in 29 of the total 110 LEAFY sequences. Because the infra-genomic polymorphisms in nuclear DNA (nrDNA) were commonly used to identify lineage hybridizations, we treated these individuals with infra-genomic polymorphisms in the chromatograms as heterozygotes. All new identified sequences were deposited in GenBank databases under accession numbers KM405789-KM 405792.

\subsection{Data analysis}

Each locus of infra-genomic polymorphisms in nrITS region appeared in every individuals of Malus sieversii, which can not provide effective information for genetic analysis. Thus, we used all of genetic analysis based on the intron 1 of the LEAFY gene. For understanding levels of genetic diversity in the sampled populations of Malus sieversii, we assessed two indices, nucleotide diversity $(\pi)$ and unbiased ex- 
pected heterozygosity $\left(\mathrm{H}_{\mathrm{e}}\right)$. Nucleotide diversity $(\pi)$ was computed by Arlequin version 3.11 (Excoffier et al., 2005). We calculated the unbiased $\mathrm{H}_{\mathrm{e}}$ based on the data matrices of alleles using GenAlEx 6.5 (Peakall and Smouse, 2012).

We assessed genetic differentiation and tested presence of phylogeographical signal for all populations of Malus sieversii by calculating $F_{\mathrm{ST}}$ (Wright's fixation index) and comparing this index with $N_{\text {ST. }} F_{\text {ST }}$ is a measure of genetic differentiation based on genotype frequencies; analogously, $N_{\mathrm{ST}}$ measures genetic differentiation based on the genetic distance between genotypes. Phylogeographical structure is suggested when $N_{\mathrm{ST}}$ is larger than $F_{\mathrm{ST}}$ (Pons and Petit, 1996). Allele distance matrix was computed in program PAUP* v.4.0b10 (Swofford, 2002) with the character of mean differences among alleles. The values of these two indices and their significances were computed and tested by constructing the distribution of the null hypothesis by means of 999 random permutations of individuals among populations using SPAGeDi 1.3 (Hardy and Vekemans, 2002).

Based on the geographical distribution of sampled populations, we divided these 15 populations into two groups (Table 1; Fig. 1): western mountains of the Junggar Basin (including EM-MFG, EM-DN, EM-GXG, EM-YZG, EM-SMG, TL-GZG1, TL-GZG2 and TL- GZG3) and the Yili Valley (including XY-JTH1, XY- JTH2, GL-MHE1, GL-SW, GL-MHE2, HC-GZG and HC-ET). We used AMOVA in the program Arlequin version 3.11 (Excoffier et al., 2005) to estimate genetic variation within populations, among populations within groups, and between two groups, and carried out significance tests based on 1,000 permutations. We also carried out pairwise mismatch distribution analysis in Arlequin version 3.11 (Excoffier et al., 2005) to test historical demographic expansion of these 15 populations. A unimodal shape in the mismatch distribution shows demographic expansion of populations, whereas stable populations should have a bi- or multi-modal distribution.

We used species distribution modeling (SDM) in this study to understand the effect of the Quaternary glacial history on the distribution pattern of Malus sieversii. We modeled the potential distributions of this species for both present climatic conditions and the Last Glacial Maximum (LGM; ca. 21 ka before present) climate scenarios based on the Model for Interdisciplinary Research on Climate (MIROC; Hasumi and Emori, 2004). Our previous studies showed that the LGM climate scenarios based on the Community Climate System Model (CCSM; Collins et al., 2006) was not suitable to model the distribution of species in this region (Zhang and Zhang, 2012; Zhang et al., 2013). We developed the present and the Last Glacial Maximum species distribution modeling of Malus sieversii based on two set of climatic data, including 19 bioclimatic variables, at a 2.5 arcmin resolution, from the WorldClim database (available at http://www. worldclim.org/download). In total, 32 occurrence points for Malus sieversii in the Yili Valley of Tianshan Mountains and the western mountains of the Junggar Basin were used in the modeling analyses, which obtained from the Chinese Virtual Herbarium (CVH; http://beta.cvh. org.cn/cms/) and our field collections. Potential distributions were modeled using the maximum entropy algorithm as implemented in MAXENT 3.3.1 (Phillips et al., 2006). The LGM potential distributions were modeled on the present climate layers with the same resolution (2.5 arcmin). Goodness of fit between the model and training data was assessed by analyzing the area under the receiver operating characteristic curve (AUC). Finally, a jackknife test was performed to measure the relative importance of climatic variables on the occurrence prediction for every distribution model.

\section{Results}

\subsection{Genetic diversity and genetic structure}

The aligned sequences for the intron 1 of the LEAFY gene were $1,074 \mathrm{bp}$ in length. A nucleotide variation was detected in this data matrix, with two types of homozygote and a heterozygote (Table 1; Fig. 1). Alleles $1 / 1$ occurred in all of 15 populations, while alleles $2 / 2$ were shown in 9 of these 15 sampled populations (Fig. 1). The heterozygote (alleles $1 / 2$ ) has wide distribution in 11 of these 15 sampled populations (Fig. 1). The length of aligned sequences for nrITS was 591 $\mathrm{bp}$, all of which include the same infra-genomic polymorphisms. Thus, phylogenetic information can not be acquired from these nrITS sequences.

In the basis of the genetic variation in the intron 1 of the LEAFY gene, the unbiased, expected hetero- 
zygosity $\left(\mathrm{H}_{\mathrm{e}}\right)$ and the nucleotide diversity $(\pi)$ in 15 populations of Malus sieversii were 0.386 and 0.000169 , respectively (Table 2 ). Significant phylogeographic structure was not detected in these 15 populations $\left(N_{\mathrm{ST}}=0.0099, F_{\mathrm{ST}}=0.0858\right.$; Table 2$)$. The AMOVA results showed that a larger proportion of the genetic differentiation distributed within populations (78.11\%; Table 3$)$. The pairwise mismatch distribution of 15 populations in Malus sieversii was unimodal (Fig. 2), indicating a sudden expansion model. Similarly, raggedness index (HRag) value under the sudden expansion model (HRag $=0.438$, $P=0.370$ ) did not reject an expansion event.

\subsection{Current and LGM species distributions}

The AUC values for the training and test data of $M a-$ lus sieversii in the present-day distribution model were $0.965 / 0.972$, suggesting a good performance for the species distribution modeling. The bioclimatic variables that contributed most to the distribution modeling of Malus sieversii were: precipitation of wettest quarter $(29.9 \%)$, precipitation of warmest quarter $(27.8 \%)$, annual mean temperature (16.4\%), isothermality (10.8\%). The present-day and LGM modeled distributions of Malus sieversii were shown in Fig. 3. The present potential distribution appeared in the Tianshan Mountains around the Yili Valley and the western mountains of the Junggar Basin. Modeling distribution based on the LGM climate scenario, as compared to that under present-day climate condition, suggests a range expansion for Malus sieversii during the interglacial period. The distribution of this species in the western mountains of the Junggar Basin

Table 2 Estimates of unbiased expected heterozygosity $\left(\mathrm{H}_{\mathrm{e}}\right)$, nucleotide diversity $(\pi)$ and two genetic differentiation indices $\left(F_{\mathrm{ST}}, N_{\mathrm{ST}}\right)$ in Malus sieversii based on the intron 1 of the LEAFY gene

\begin{tabular}{ccccccc}
\hline$N_{\text {pop }}$ & $N_{\text {ind }}$ & $\mathrm{A}$ & $\mathrm{H}_{\mathrm{e}}$ & $\pi$ & $F_{\mathrm{ST}}$ & \\
\hline 15 & 110 & 2 & $0.386 \pm 0.049$ & $\begin{array}{c}0.000169 \pm \\
0.000244\end{array}$ & 0.0858 \\
\hline
\end{tabular}

Notes: Numbers of populations $\left(N_{\text {pop }}\right)$, sequenced individuals $\left(N_{\text {ind }}\right)$ and alleles $(\mathrm{A})$ in the intron 1 of the LEAFY gene were shown. Data are means \pm SD.

Table 3 Results of analysis of molecular variance (AMOVA) of ribotype frequencies for populations and population groups of Malus sieversii

\begin{tabular}{lccccc}
\hline Source of variation & $d f$ & SS & VC & PV $(\%)$ & Fixation index \\
\hline Among groups & 1 & 1.223 & 0.02085 & 20.59 & $F_{\mathrm{CT}}=0.20586$ \\
Among populations within groups & 13 & 1.151 & 0.00132 & $F_{\mathrm{SC}}=0.01641$ \\
Within populations & 95 & 7.516 & 0.07912 & 78.11 \\
Total & 109 & 9.891 & 0.10129 & $F_{\mathrm{ST}}=0.21889$ \\
\hline
\end{tabular}

Notes: $d f$, degree of freedom; SS, sum of squares; $\mathrm{VC}$, variance component; PV, percentage of variation; $F_{\mathrm{CT}}$, correlation of chlorotypes within groups relative to total; $F_{\mathrm{SC}}$, correlation within populations relative to groups; $F_{\mathrm{ST}}$, correlation within populations relative to total.

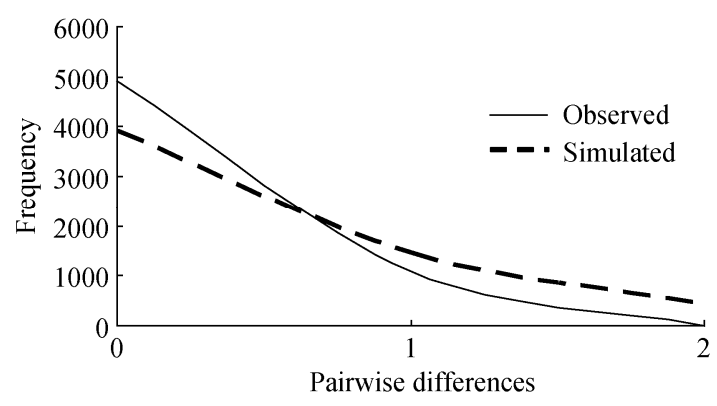

Fig. 2 Pairwise mismatch distributions of nrDNA genotypes for populations of Malus sieversii in the Yili Valley and the western mountains of the Junggar Basin

disappeared during the LGM period. Meanwhile, the LGM distribution was greatly diminished in the Tianshan Mountains around the Yili Valley.

\section{Discussion and conclusion}

\subsection{Genetic structure of Malus sieversii}

In this study, we sampled 15 populations from Xinjiang, the eastern range of Malus sieversii. Based on the nucleotide variation in the intron 1 of the LEAFY gene, these 15 populations had low level of genetic diversity $\left(\mathrm{H}_{\mathrm{e}}=0.386, \pi=0.000169\right.$; Table 2). Using SSR and SRAP makers, Zhang et al. (2007, 2009) also found that populations of Malus sieversii from Xinjiang had low level of genetic diversity (SSR: Nei's gene diversity index $=0.2619$; SRAP: average panmictic heterozygosity $=0.3359$ ). In addition, we found 


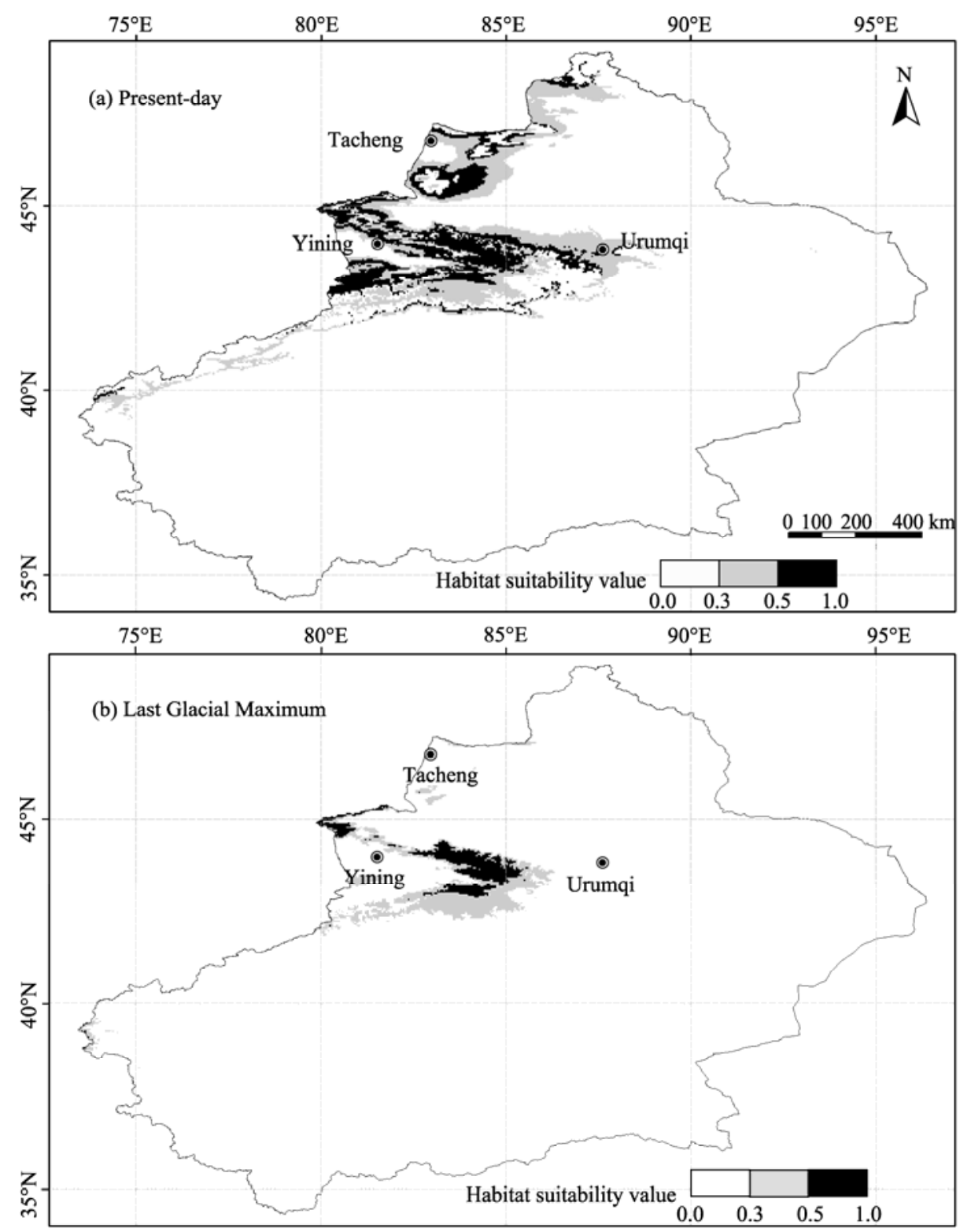

Fig. 3 The present-day (a) and the Last Glacial Maximum (LGM; b) distributions of Malus sieversii in the Yili Valley and the western mountains of the Junggar Basin as derived from the species distribution modeling in MAXENT 3.3.1

the level of genetic differentiation was much low in the populations of Malus sieversii from Xinjiang $\left(F_{\mathrm{ST}}=0.0858\right.$; Table 2$)$. The molecular variances of these 15 populations were larger distributed within populations than between populations $(78.1 \%$ vs $1.3 \%$; Table 3). It is well consistent with the results from previous studies based on different types of molecular markers (Zhang et al., 2007; Yan et al., 2008; Zhang et al., 2009). In the western range of this species, populations of Malus sieversii in Kyrgyzstan showed a low level of genetic differentiation among sampling sites $\left(F_{\mathrm{st}}=0.05\right.$; Richards et al., 2009). The genetic varia- tions of Malus sieversii in Kyrgyzstan were also largely distributed within populations $(85 \%$; Lamboy et al., 1996).

In the population level, these eight north populations in the western mountains of the Junggar Basin had lower level of genetic diversity than the seven south populations in the Yili Valley (0.308 vs. 0.488 ; Table 1). Similarly, the data of genetic diversity in populations from Kyrgyzstan indicated that the southwestern populations were more admixed and more diverse than the northern regions (Richards et al., 2009). In terms of the distribution of alleles, the het- 
erozygous genotype of alleles $1 / 2$ appeared in most of these 15 populations (Table1; Fig. 1). This indicates hybridization of Malus sieversii could be widespread in the eastern distribution.

\subsection{Effect of the Quaternary glaciations on the historical demography of Malus sieversii}

Wild fruit forest in Xinjiang is a valuable "relict" of broad-leaved forest in the Central Asian mountain region (Zhang, 1973). In the Central Asian mountains, the Quaternary glaciations have been well developed (Xu et al., 2010) and intensively influenced the flora of this mountain region (Wen and Shi, 1993). With respect to the mountain plants, the glacial refugia have served as a gene pool and reserved high levels of genetic diversity during the cold glacial periods. And then, these species could have experienced a demographic expansion from the glacial refugia during the warm interglacial epochs (Zhang and Zhang, 2012; Zhang et al., 2013). Malus sieversii, a major component of wild fruit forest in the Central Asian Mountains, could be also responded to these glacial-interglacial climatic changes. From the spatial genetic structure of Malus sieversii, one of the homozygous genotypes (alleles 1/1) existed in each population (Fig. 1). It means the founder effect could be prevailing during the interglacial warming in the eastern range of Malus sieversii. Mismatch distribution of genetic variations also revealed that these sampled populations could have undergone a demographic expansion during the course of historical evolution. Meanwhile, these 15 populations didn't display a significant phylogeographic structure. This incomplete lineage sorting could result from recent demographic expansions. These seven southern populations in the Yili Valley with high level of genetic diversity (Table 1) could serve as the refugia in the eastern range of Malus sieversii. These eight northern populations in the western mountains of the Junggar Basin might migrate from the refugial region during the interglacial expansion (Fig. 3). Hybridization of two different lineages could result from early glaciations before the latest interglacial expansion due to the wide distribution of heterozygote (alleles 1/2) in Malus sieversii (Fig. 1).

\subsection{Implications for conservation}

Malus sieversii is a valuable germplasm resource to improve the domesticated apples from both genetic diversity and cultivar breeding. In the view of conservation genetics, populations with high levels of genetic diversity and allele diversity should be treated as priority protected areas. However, the spatial genetic structure of Malus sieversii didn't show any populations have particular alleles (Fig. 1) based on the intron 1 of the LEAFY gene. It would be difficult to recognize the locations of priority protected areas. In the distribution modeling of Malus sieversii (Fig. 3), populations in the western mountains of the Junggar Basin had high sensibility to disturbance, such as climatic changes. Populations in the Yili Valley have served as glacial refugia, and could provide safe harbors for Malus sieversii to resist disturbances. Thus, the populations in the western mountains of the Junggar Basin should be paid more attentions to be protected. At the same time, the germplasm resource of Malus sieversii should be protected taking the different phenotypes of fruits into account.

\section{Acknowledgements}

This research was supported by the National Basic Research Special Program of China (2012FY111500), the Innovation Research Group Program of Chinese Academy of Sciences and State Administration of Foreign Experts Affairs of China (KZCX2-YW-T09), the State International Science and Technology Cooperation Program of China (2010DFA92720) and the Program of the Xinjiang Institute of Ecology and Geography, Chinese Academy of Sciences. We greatly thank Mr. KaiQing XIE in the Shihezi University, Xinjiang, and Mr. Zheng XU in the Yili Institute of Horticulture Research, Xinjiang, for their helps to materials collection.

\section{References}

Collins W D, Bitz C M, Blackmon M L, et al. 2006. The community climate system model version 3 (CCSM3). Journal of Climate, 19: 2122-2143.

Doyle J J, Doyle J L. 1987. A rapid DNA isolation procedure for small quantities of fresh leaf tissue. Phytochemical Bulletin, 19: 11-15.

Excoffier L, Laval G, Schneider S. 2005. Arlequin (version 3.0): an integrated software package for population genetics data analysis. Evolutionary Bioinformatics Online, 1: 47-50.

Hardy O J, Vekemans X. 2002. SPAGeDi: a versatile computer program to analyse spatial genetic structure at the individual or population levels. Molecular Ecology Notes, 2: 618-620.

Harris S A, Robinson J P, Juniper B E. 2002. Genetic clues to the origin of the apple. Trends in Genetics, 18: 426-430.

Hasumi H, Emori S. 2004. K-1 Coupled GCM (MIROC) Description. Tokyo: Center for Climate System Research, University of Tokyo. 
Hou B, Xu Z. 2005. Relationship of the occurences and evolutions of wild-fruit forests with climatic factors in the Tianshan Mountain. Acta Botanica Boreali-Occidentalia Sinica, 25: 2266-2271. (in Chinese)

Lamboy W F, Yu J, Forsline P L, et al. 1996. Partitioning of allozyme diversity in wild populations of Malus sieversii L. and implications for germplasm collection. Journal of the American Society for Horticultural Science, 121: 982-987.

Lo E Y, Stefanović S, Christensen K I, et al. 2009. Evidence for genetic association between East Asian and western North American Crataegus L. (Rosaceae) and rapid divergence of the eastern North American lineages based on multiple DNA sequences. Molecular Phylogenetics and Evolution, 51: 157-168.

Peakall R, Smouse P E. 2012. GenAlEx 6.5: genetic analysis in Excel. Population genetic software for teaching and research-an update. Bioinformatics, 28: 2537-2539.

Phillips S J, Anderson R P, Schapire R E. 2006. Maximum entropy modeling of species geographic distributions. Ecological Modelling, 190: 231-259.

Pons O, Petit R. 1996. Measwring and Testing Genetic Differentiation With Ordered Versus Unordered Alleles. Genetics, 144: 1237-1245.

Richards C M, Volk G M, Reilley A A, et al. 2009. Genetic diversity and population structure in Malus sieversii, a wild progenitor species of domesticated apple. Tree Genetics \& Genomes, 5: 339-347.

Robinson J, Harris S, Juniper B. 2001. Taxonomy of the genus Malus Mill. (Rosaceae) with emphasis on the cultivated apple, Malus domestica Borkh. Plant Systematics and Evolution, 226: 35-58.

Shaw J, Lichey E B, Beck J T, et al. 2005. The tortoise and the hare II: relative utility of 21 noncoding chloroplast DNA sequences for phylogenetic analysis. American Journal of Botany, 92: 142-166.

Shaw J, Lichey E B, Schilling E E, et al. 2007. Comparison of whole chloroplast geneome sequences to choose noncoding regions for phylogenetic studies in angiosperms: the tortoise and the hare III. American Journal of Botany, 94: 275-288.

Sitpaeva G, Chekalin S. 2013. Forestry activity is one mechanism of invasion by arboreal plants. Journal of Arid Land, 5: 434-438.
Swofford D L. 2002. PAUP*. Phylogenetic Analysis Using Parsimony (* and Other Methods). Version 4. Sunderland, MA, USA: Sinauer Associates.

Thompson J D, Gibson T J, Plewniak F, et al. 1997. The CLUSTAL_X windows interface: flexible strategies for multiple sequence alignment aided by quality analysis tools. Nucleic Acids Research, 25: 4876-4882.

Wen Q Z, Shi Y F. 1993. The Quaternary climo-environment changes in Chaiwopu Basin of Xinjiang region. Chinese Geographical Science, 3: $147-158$.

Xu X, Kleidon A, Miller L, et al. 2010. Late Quaternary glaciation in the Tianshan and implications for palaeoclimatic change: a review. Boreas, 39: 215-232.

Yan G, Long H, Song W, et al. 2008. Genetic polymorphism of Malus sieversii populations in Xinjiang, China. Genetic Resources and Crop Evolution, 55: 171-181.

Zhang C, Chen X, He T, et al. 2007. Genetic structure of Malus sieversii population from Xinjiang, China, revealed by SSR markers. Journal of Genetics and Genomics, 34: 947-955.

Zhang C, Chen X, Lin Q, et al. 2009. SRAP markers for population genetic structure and genetic diversity in Malus sieversii from Xinjiang, China. Acta Horticulturae Sinica, 36: 7-14. (in Chinese)

Zhang H X, Zhang M L. 2012. Genetic structure of the Delphinium naviculare species group tracks Pleistocene climatic oscillations in the Tianshan Mountains, arid Central Asia. Palaeogeography, Palaeoclimatology, Palaeoecology, 353-355: 93-103.

Zhang H X, Zhang M L, Sanderson S C. 2013. Retreating or standing: responses of forest species and steppe species to climate change in arid eastern Central Asia. PloS One, 8: e61954.

Zhang X S. 1973. Study on the ecological and geographical characteristics and issues of community science of the wild fruit forests in the Yili Valley. Acta Botanica Sinica, 15: 239-253. (in Chinese)

Zhou Z, Li Y. 2000. The RAPD evidence for the phylogenetic relationship of the closely related species of cultivated apple. Genetic Resources and Crop Evolution, 47: 353-357. 